\title{
Lysozyme and mucolytics - the hidden allergen
}

Singapore Med J 2020; 61(9): 497 https://doi.org/10.11622/smedj.2020132

Dear Sir,

We herein report a case of a young girl who developed an adverse drug reaction (ADR) to a commonly prescribed lysozyme-containing mucolytic. Our patient had a background of infantile atopic dermatitis, (cow's) milk anaphylaxis and egg allergy. At three years of age, she visited her family doctor for an upper respiratory tract infection and was prescribed Leftose ${ }^{\circledR}$, a lysozyme-containing product, for the first time. Within 30 minutes of Leftose ingestion, she developed generalised urticaria. There were no systemic complaints. She was given oral antihistamines and monitored in the hospital for one day.

A subsequent prick-to-prick test to the Leftose syrup confirmed immediate hypersensitivity with wheal size of $14 \mathrm{~mm}$. A skin prick test to egg white extract was positive (wheal size $9 \mathrm{~mm}$ ), and serum-specific immunoglobulin E to egg was (also positive at) $36.30 \mathrm{kU} / \mathrm{L}$.

Lysozyme ( $\mathrm{N}$-acetyl muramidase) is an enzyme with bactericidal activity that has purported expectorant and mucolytic benefits. Since 1989, lysozyme-containing products have been used by Singaporean doctors and are available over the counter. However, evidence from the published literature has failed to show the efficacy of lysozyme compared to a placebo. In June 2017, the Health Sciences Authority (HSA), Singapore, announced the phasing out of lysozyme-containing products as therapeutic products by 31 December 2018. ${ }^{(1)}$ Nonetheless, this does not preclude the usage of lysozyme as health supplements.

Lysozyme is derived from hen's egg white or through biofermentation. Eggs contain 3\% lysozyme, which has been characterised as an allergen (Gal d 4). Up to $32 \%$ of individuals with egg allergy are sensitised to lysozyme. ${ }^{(2)}$ Drugs containing lysozyme have been known to cause the allergic reactions of urticaria, angio-oedema and even anaphylaxis. There is also a case report of a lysozymecontaining product causing toxic epidermal necrolysis. ${ }^{(3)}$ Most of these patients were previously sensitised or allergic to eggs, while in others, lysozyme was reported to be the primary sensitiser. Within our clinical practice in a tertiary paediatric allergy unit, parents of egg-allergic children are taught how to read ingredient lists and avoid products such as lysozyme in food and drugs. Unfortunately, ADRs to lysozyme-containing products still occur.

As the prevalence of food allergy rises, so does the risk of hypersensitivity to food additives in drugs and health products. Physicians should exercise caution when prescribing lysozyme-containing products in patients with egg allergy because of the risk of a lifethreatening reaction. The HSA is working with companies marketing lysozyme-containing products to update product information labels on this safety information. ${ }^{(4)}$ In our unit, we have prepared a written letter regarding lysozyme avoidance for parents of children with egg allergy to present to their physicians. We are working as a community to raise awareness and improve the safety of our patients with egg allergy.

Yours sincerely,

Lynette $\underline{T a n}^{1}$, Kok Wee $\underline{\text { Chong }}^{1}$, Si Hui Goh ${ }^{1}$

${ }^{1}$ Allergy Service, Department of Paediatric Medicine, KK Women's and Children's Hospital, Singapore. lynette.tan.Il@singhealth.com.sg

\section{References}

1. Health Sciences Authority, Singapore. HSA updates on the phasing-out of lysozyme containing products as therapeutic products. Available at: https://www.hsa.gov. sg/docs/default-source/announcements-csg/hsa-updates/hsaupdates_phasing-out-of-lysozyme-containing-products.pdf. Accessed September $14,2020$.

2. Audicana Berasategui MT, Barasona Villarejo MJ, Corominas Sánchez M, et al. Potential hypersensitivity due to the food or food additive content of medicinal products in Spain. J Investig Allergol Clin Immunol 2011; 21:496-506.

3. Kobayashi M, Yamamoto O, Yasuda H, Asahi M. A case of toxic epidermal necrolysis-type drug eruption induced by oral lysozyme chloride. J Dermatol 2000; 27:401-4.

4. Health Sciences Authority, Singapore. Caution on the use of lysozyme-containing products in patients with known egg allergy. Available at: https://www.hsa.gov.sg/ docs/default-source/announcements/adverse-drug-reaction-news-bulletin/adr_news_may2018_vol20_no1.pdf. Accessed September 14, 2020. 